\title{
THE PLASTIC INDENTATION OF A LAYER BY A FLAT PUNCH*
}

\author{
BY \\ R. T. SHIELD 1 \\ Fort Halstead, Kent, England
}

Summary. Upper and lower bounds for the average pressure in the indentation by a flat, smooth punch of the plane surface of a layer of elastic-perfectly plastic material resting on a rough, rigid base are obtained by the application of the limit-design theorems. The material of the layer is assumed to obey Tresca's yield criterion of constant maximum shearing stress during plastic deformation. The square punch problem is considered in detail for lavers whose thickness is greater than one-fourteenth of the width of the punch. For thinner layers, reasonably close upper and lower bounds for the average pressure over the square punch are obtained as functions of the relative thickness of the layer. The circular punch is considered briefly, and the bounds obtained determine the indentation force with sufficient accuracy for layers which are not too thick compared with the width of the punch.

1. Introduction. The problem under consideration is the plastic indentation by a flat smooth punch of the plane surface of a layer of elastic-perfectly plastic material resting on a rough rigid base, Fig. 1 . A complete solution to the problem under conditions of

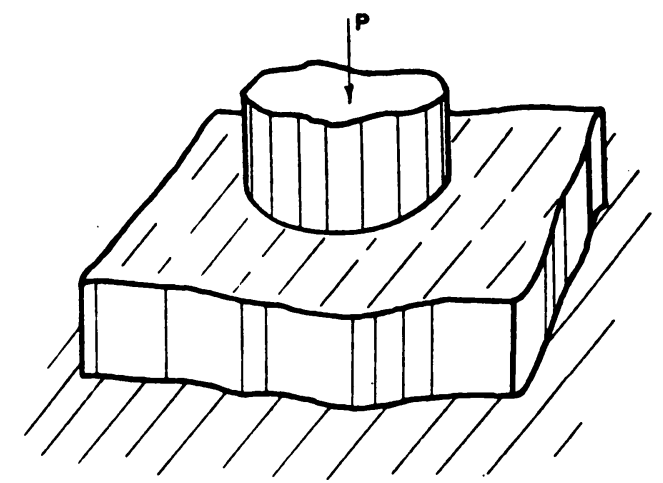

FIG. 1. Rigid punch on layer of material resting on rough rigid base.

plane strain has been obtained by Wang and Lee [1] ${ }^{2}$. The three dimensional punch problem is one of considerable difficulty at the present stage of elastic-plastic analysis. However, as in previous work [2] on the indentation of an infinitely thick layer, the limit-design theorems $[3,4,5]$ can be used to determine upper and lower bounds for the indentation force during the incipient plastic yielding of the layer.

In the following work the material of the layer is assumed to obey Tresca's yield criterion of constant maximum shearing force $k$ during plastic deformation. This yield condition approximates to the behaviour of a frictionless cohesive soil such as clay. The square punch is considered first and the bounds obtained for the average pressure

*Received March 19, 1954.

'Senior Research Fellow, Armament Research Establishment.

"Numbers in square brackets refer to the Bibliography at the end of the paper. 
on the punch for layers of varying thickness determine the indentation force with sufficient accuracy for practical purposes. The lower bound $5 k$ for the punch pressure obtained in [2] for a square or rectangular punch on a layer of infinite thickness is improved to the plane strain value $(2+\pi) k$. Finally, bounds are obtained for a circular punch, and these bounds are in close agreement for layers which are thin compared with the width of the punch. The stress field used to obtain lower bounds for the circular punch pressure can be adapted to provide lower bounds for any convex area of indentation.

2. Limit-design theorems. The limit-design theorems developed recently $[3,4,5]$ provide a general method for determining the point at which collapse will occur in assemblages of rigid and of elastic-perfectly plastic bodies. Collapse is used here to describe conditions under which plastic flow would occur under constant loads if the accompanying changes in the geometries of the bodies were disregarded. We now outline the limitdesign theorems in the form required in the following work.

The boundary conditions are assumed to be of the stress type, i.e. each component, $T_{x}, T_{y}$, or $T_{z}$, of the surface traction is given except where the corresponding velocity component, $V_{x}, V_{y}$, or $V_{z}$, or the corresponding relative velocity component at the interface of an assemblage is prescribed to be zero. A stress field is called statically admissible if the field satisfies the stress boundary conditions, satisfies the equations of equilibrium throughout the volume of the bodies, and if the yield condition is violated nowhere. Where the stress components are continuous functions of position, the equations of equilibrium are

$$
\frac{\partial \sigma_{x}}{\partial x}+\frac{\partial \tau_{x y}}{\partial y}+\frac{\partial \tau_{x z}}{\partial z}=0
$$

and two similar equations. The equilibrium conditions across a surface of discontinuity in the stress field require that the expression

$$
\sigma_{x} n_{x}+\tau_{x y} n_{y}+\tau_{x z} n_{z}
$$

and each of the two similar expressions, must have the same value whether it is evaluated from the stresses on one or the other side of the discontinuity surface. In expression (2), $n_{x}, n_{y}$, and $n_{z}$ are the components of the unit normal to the surface of stress discontinuity.

From any given velocity field $V_{x}, V_{y}, V_{z}$, strain rates can be derived in the usual way

$$
\dot{\epsilon_{x}}=\frac{\partial V_{x}}{\partial x}, \cdots, \dot{\gamma_{x y}}=\frac{\partial V_{x}}{\partial y}+\frac{\partial V_{y}}{\partial x}, \cdots .
$$

If the strain rates are considered as purely plastic strain rates, the internal rate of dissipation of energy can be calculated. Also the external rate of dissipation of energy can be calculated as the rate at which the applied tractions do work on their points of application. A velocity field is called kinematically admissible if the velocity components $V_{x}, V_{y}, V_{z}$ satisfy the incompressibility condition

$$
\frac{\partial V_{x}}{\partial x}+\frac{\partial V_{y}}{\partial y}+\frac{\partial V_{z}}{\partial z}=0,
$$

satisfy any imposed velocity boundary condition, and if the total external rate of dissipation of energy is not less than the total internal rate of dissipation of energy. 
The limit-design theorems for the case of all surface tractions increasing in proportion have been formulated as follows:

Theorem 1. Collapse will not occur until the largest values of the surface tractions are reached for which it is possible to find a statically admissible stress field.

Theorem 2. Collapse will occur under the smallest values of the surface tractions for which it is possible to find a kinematically admissible velocity field.

With these two theorems, lower and upper bounds can be obtained for the collapse values of the applied surface tractions.

Since Tresca's criterion of constant maximum shearing stress is used in the following work, statically admissible stress fields must nowhere involve a shearing stress greater than the value $k$. Also, for this material, the internal rate of dissipation of energy due to a plastic strain rate is given by [2] as

$$
D=2 k \max \left|\epsilon^{\cdot}\right| \text {, }
$$

where $\max \left|\cdot \epsilon^{\cdot}\right|$ denotes the absolute value of the numerically greatest principal component of the plastic strain rate. It now follows from the definition of a kinematically admissible velocity field $V_{x}, V_{y}, V_{z}$, that the velocity field must satisfy the inequality

$$
\int_{S}\left(T_{x} V_{x}+T_{y} V_{y}+T_{z} V_{z}\right) d S \geq \int_{D} 2 k \max \left|\epsilon^{\cdot}\right| d V,
$$

where $S$ is the bounding surface and $V$ the volume of the body or assemblage of bodies, and where $\max \left|\epsilon^{\circ}\right|$ denotes the absolutely largest principal component of the derived plastic strain rate. The strain rate is zero for a rigid body so that rigid bodies in the assemblage contribute nothing to the right hand side of the inequality (6). Discontinuities in the tangential velocity are permissible in kinematically admissible velocity fields. For each surface of discontinuity $S_{D}$ in the field, the expression

$$
\int_{S_{D}} k \Delta V d S_{D}
$$

must be added to the right hand side of (6), where $\Delta V$ is the magnitude of the relative change in velocity across the surface $S_{D}$.

3. Statement of the problem. We are considering the indentation of the upper plane surface of an infinite layer of perfectly plastic material by a flat-ended rigid punch or footing. The lower plane surface of the layer is parallel to the upper surface and rests on a perfectly rough, rigid base (or equivalently, there is complete attachment between the base and material in the layer). The indenting surface of the punch is smooth and it is assumed that the free end of the punch is normally and centrally loaded. The force $P$ on the punch (Fig. 1) is increased until indentation occurs due to plastic yielding of the material in the layer. The punch, the layer and the base constitute an assemblage of bodies to which the limit-design theorems are applicable. Statically admissible stress fields for this problem must be such that the pressure between the punch and the material is normal to the plane surface. Also the resultant normal force must act through the centroid of the area of contact, but the distribution of the normal pressure is not restricted in any other way, since the material of the punch is rigid. At the interface of the layer and the base, the tractions may have any value compatible with statically admissible stress fields in the material of the layer, since the base is rigid and is perfectly rough. 
The rigidity of the punch implies that kinematically admissible velocity fields must be such that the area of indentation moves as a plane surface, although there may be slip between the material and the punch, which is smooth. The material immediately adjacent to the rough base must not move relative to the base. However, a surface of velocity discontinuity between the material and the material immediately adjacent to the base is permissible.

4. Square punch. Upper bounds for the indentation pressure. Upper and lower bounds for the indentation pressure have been obtained previously [2] for the indentation of an infinitely thick layer by a square punch. It was shown that the average pressure over the square area of indentation lies between the values $5 k$ and $5.71 k$. The kinematically admissible velocity field used in [2] to obtain the upper bound $5.71 k$ involved nonzero velocities in a region of the material of the layer which extended to a depth $a \sin 34^{\circ}$ below the surface of the material, where $2 a$ is the width of the punch. It follows that $5.71 k$ is also an upper bound for the average pressure in the indentation of a layer of finite thickness $h$, provided that the thickness of the layer is such that

$$
\frac{a}{h} \leq \operatorname{cosec} 34^{\circ}=1.79 .
$$

For layers which are such that $a / h$ is greater than 1.79 , the velocity field can be modified in an obvious manner so that it lies wholly within the material of the layer and can therefore be used to provide upper bounds for the average indentation pressure. The relevant formulae are in fact given in [2] so that, in the interests of brevity, only the results of carrying out this procedure will be given here. It is found that the values $5.73 k, 5.86 k, 6.10 k$ are upper bounds for the average identation pressure when $a / h$ has the values $2,2.5,3$ respectively. These values of the upper bound $p_{u}$ are plotted against $a / h$ in Fig. 2, and are joined by a smooth curve, which is marked I in Fig. 2, to the line $p_{u}=5.71 k(a / h<1.79)$.

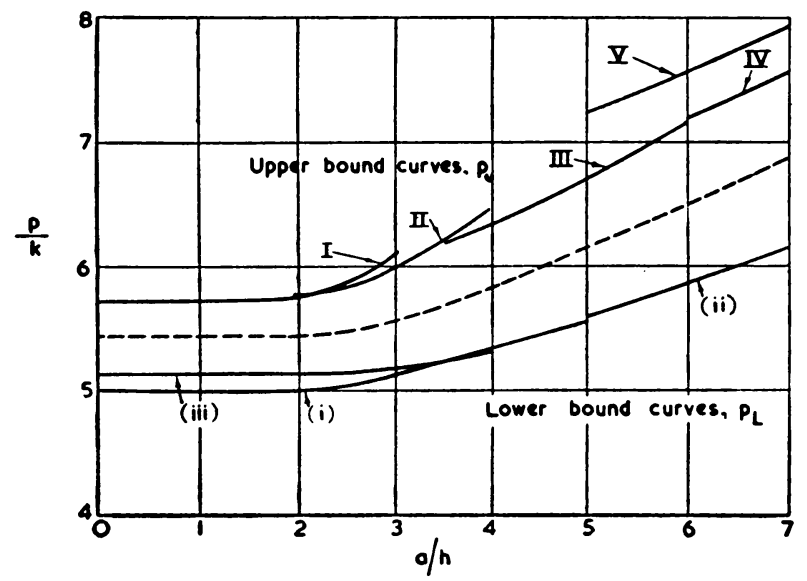

FIG. 2. Upper and lower bounds for the average pressure over the square punch.

For values of $a / h$ greater than 2.3 approximately, a better upper bound is obtained by means of a velocity field which is somewhat similar to the velocity field of [2] mentioned above. The velocity field has two vertical planes of symmetry which pass through 
the mid-points of opposite sides of the square area of indentation. Hence we need only consider that part of the velocity field which accommodates the downward motion of one-fourth of the total area of contact. This portion of the velocity field is shown diagrammatically in Fig. 3. The point $B$ in Fig. 3 is a corner of the square area of contact
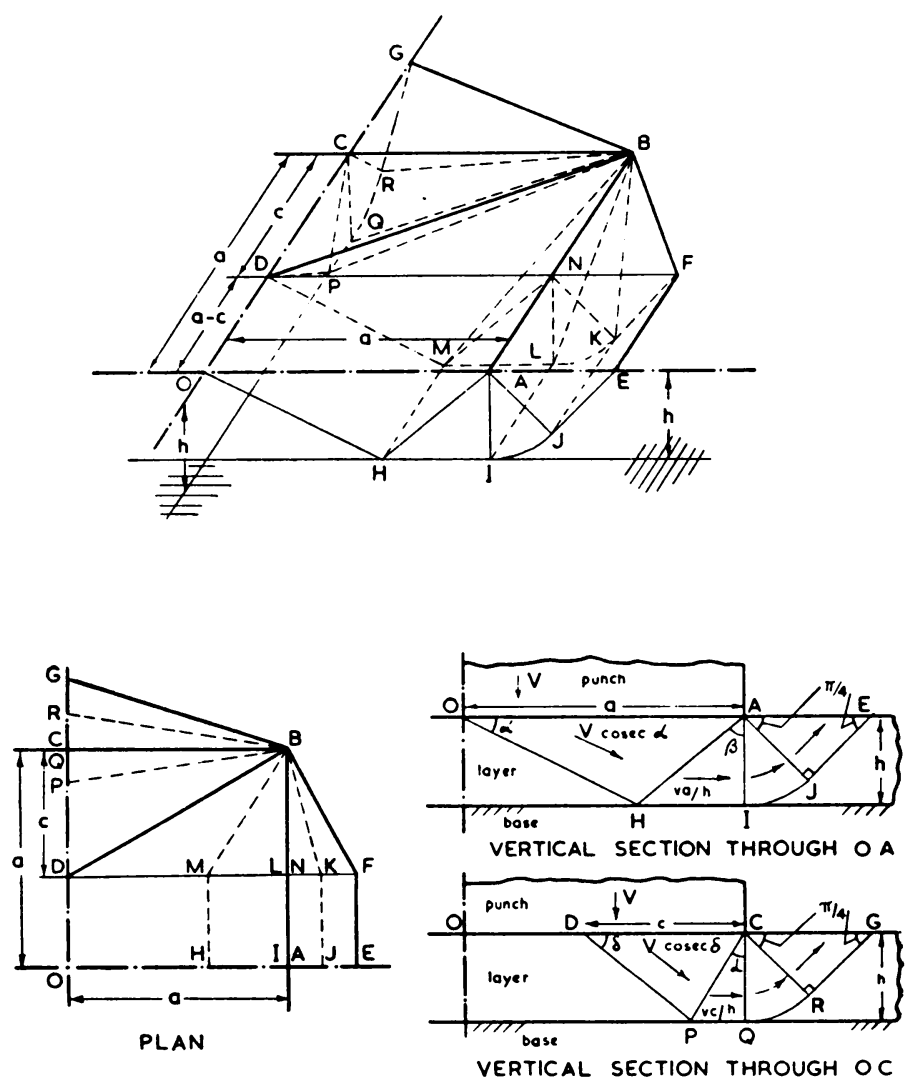

Fig. 3. Velocity field for square punch on layer.

between the punch and the layer and $A, C$ are the mid-points of the two sides of the square which meet at $B ; O$ is the centre of the square. The part of the velocity field accommodating the downward motion of the area $O A B C$ involves flow in the volumes $B D O E J I H M L K F$ and $B G D P Q R$, which we shall call volumes 1 and 2 respectively.

Volume 1 consists of tetrahedra $B D M N, B N M L, B N K F$, a section of a right circular cone $B N L K$, triangular prisms DMNOHA, NMLAHI, NKFAJE, and a section of a right circular cylinder $N L K A I J$. Vertical sections of volume 1 by planes parallel to the line $O A$ are geometrically similar to the section through $O A$ but vary in size for those sections which cut the line $B N$. The rectangle $M L I H$ is at a distance $h$ below the upper surface of the layer of material. Angles $A O H, H A I$ are denoted by $\alpha, \beta$ respectively, while angles $J A E, A E J$ are both 45 degrees. The region $B D M N O H A$ moves as a rigid body with velocity $v \operatorname{cosec} \alpha$ in the direction $O H$, where $v$ is the initial downward velocity of the punch (i.e. the downward velocity of the area $O A B C$ ). In the region $B N M L K F A H I J E$, the streamlines of the velocity field are parallel to the line $H I J E$ 
and the velocity along each streamline has the constant value $v a / h$. This value of the velocity is obtained from the condition that the relative velocity between the regions on either side of the velocity discontinuity surface $B M H A N$ must lie in the plane $B M H A N$.

In volume 2 , the tetrahedron $B D P C$ moves as a rigid body with velocity $v \operatorname{cosec} \delta$ in the direction $D P$, where $\delta$ is the angle $C D P$. In the remainder of volume 2, which consists of two tetrahedra and a section of a right circular cone, the streamlines of the field are parallel to the line $D P Q R G$ and the velocity is $v c / h, C D$ and $C Q$ being of lengths $c$ and $h$ respectively.

Energy is dissipated in the discontinuity surfaces between the material at rest and the material flowing in volumes 1 and 2 , and in the discontinuity surfaces $B M H A N$, $B C P$. (The rectangle $M L I H$ is a surface of velocity discontinuity between the material in the prism NMLAHI and the material lying in the lower surface of the layer immediately adjacent to the base). Energy is also dissipated in the cylindrical and conical regions where the plastic strain rate is non-zero. With expressions (5) and (7), it is not difficult to calculate the total rate of internal dissipation of energy. It is found that the rate at which work is done by the force on the punch will not be less than the internal rate of dissipation of energy if the average pressure on the punch is not less than $f(c, \beta, \gamma)$, where

$$
\begin{aligned}
f(c, \beta, \gamma) / k= & 1+\frac{\pi}{2}+\frac{a}{h}-\frac{c}{h}-\tan \beta+\frac{h}{a}\left(2-\frac{3}{2} \frac{c}{a}\right) \sec ^{2} \beta+\frac{1}{2} \frac{h}{a} \sec ^{2} \gamma \\
& +\frac{c}{a}\left\{\tan \beta-1-\frac{\pi}{4}+\frac{1}{2}\left(1+\frac{h^{2}}{a^{2}}\right)^{1 / 2}\left(1+\frac{\pi}{4}+\tan \gamma\right)\right\} \\
& +\frac{1}{2} \frac{h}{a} \operatorname{cosec} \delta\left(\frac{c^{2}}{a^{2}}+\operatorname{cosec}^{2} \delta\right)^{1 / 2}+\frac{1}{2} \frac{h}{a} \operatorname{cosec} \alpha\left(1+\frac{c^{2}}{a^{2}} \operatorname{cosec}^{2} \alpha\right)^{1 / 2} \\
& +\frac{1}{2}\left(\frac{h^{2}}{a^{2}}+\frac{c^{2}}{a^{2}}\right)^{1 / 2}\left(1+\frac{\pi}{4}+\tan \beta\right) .
\end{aligned}
$$

In this expression $\gamma$ is the angle $P C Q$, the angles $\alpha, \delta$ being obtained from the relations $\cot \alpha+\tan \beta=a / h, \cot \delta+\tan \gamma=c / h$. It follows from Theorem 2 that the function $f(c, \beta, \gamma)$ is an upper bound for the collapse value of the average indentation pressure. Minimizing the function $f(c, \beta, \gamma)$ with respect to $c, \beta, \gamma$ provides the best upper bound for a particular value of $a / h$ which can be obtained from this velocity field. The following table gives the upper bounds $p_{u}$ obtained by approximately minimising $f(c, \beta, \gamma)$ for certain values of $a / h$, together with the values of $c, \beta, \gamma$ used in each case

$\begin{array}{rccccc}a / h: & 2 & 2.5 & 3 & 3.5 & 4 \\ p_{u} / k: & 5.77 & 5.81 & 5.98 & 6.20 & 6.47 \\ c / a: & 0.70 & 0.68 & 0.62 & 0.59 & 0.56 \\ \beta: & 24^{\circ} & 30^{\circ} & 35^{\circ} & 40^{\circ} & 44^{\circ} \\ \gamma: & 19^{\circ} & 23^{\circ} & 26^{\circ} & 27^{\circ} & 29^{\circ}\end{array}$

The values of $p_{u}$ are plotted against $a / h$ in Fig. 2 and are joined by a smooth curve (marked II in Fig. 2).

For thinner layers, $a / h>3.4$ approximately, better upper bounds are obtained with the velocity field shown diagrammatically in Fig. 4 . The field is a modification of the field of Fig. 3. As before, $O A B C$ is one-fourth of the area of indentation and its downward motion is accommodated by flow of the material in the regions shown. The region which was called volume 2 in describing the velocity field of Fig. 3 is left unchanged, while volume 1 now contains four instead of two blocks of material moving as rigid 


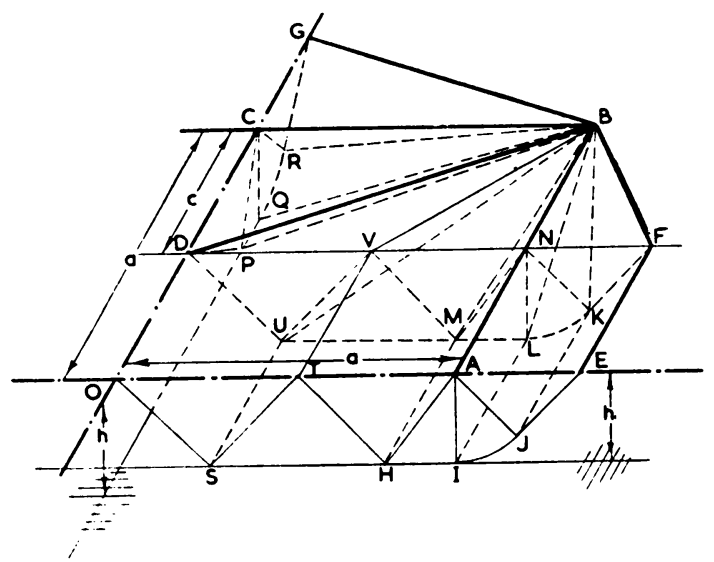

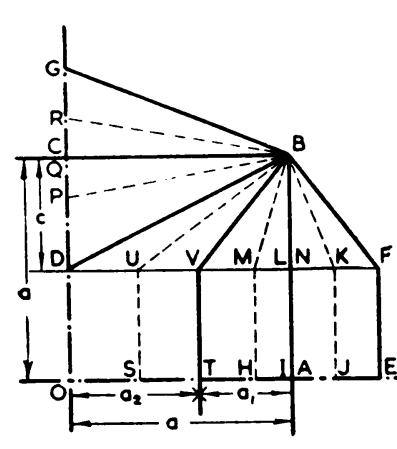

PLAN.
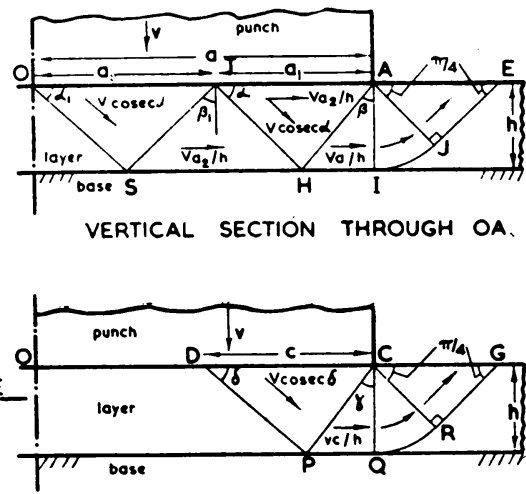

VERTICAL SECTION THROUGH OC.

FIG. 4. Modification of velocity field of Fig. 3 to thinner layers.

bodies under the area $O A B D$. The velocities in the various regions are shown in Fig. 4, and are obtained from the velocity conditions at the surface of the punch and at the various discontinuity surfaces. It is found that this velocity field will be kimematically admissible if the average pressure on the punch is not less than $g\left(c, a_{1}, \beta, \beta_{1}, \gamma\right)$, where

$$
\begin{aligned}
& g\left(c, a_{1}, \beta, \beta_{1}, \gamma\right) / k=1+\frac{\pi}{2}+\frac{1}{2}\left(1+\frac{\pi}{4}\right)\left\{\left(\frac{h^{2}}{a^{2}}+\frac{c^{2}}{a^{2}}\right)^{1 / 2}+\frac{c}{a}\left(1+\frac{h^{2}}{a^{2}}\right)^{1 / 2}-2 \frac{c}{a}\right\} \\
& +\frac{1}{2} \frac{h}{a}\left\{\operatorname{cosec} \delta\left(\frac{c^{2}}{a^{2}}+\operatorname{cosec}^{2} \delta\right)^{1 / 2}+\sec ^{2} \gamma\right\}+\frac{1}{2} \frac{c}{a} \tan \gamma\left(1+\frac{h^{2}}{a^{2}}\right)^{1 / 2} \\
& +\frac{h}{a}\left\{\left(1-\frac{c}{a}\right) \operatorname{cosec}^{2} \alpha_{1}+\frac{1}{2} \operatorname{cosec} \alpha_{1}\left(1+\frac{c^{2}}{a^{2}} \operatorname{cosec}^{2} \alpha_{1}\right)^{1 / 2}+\left(1-\frac{c}{a}\right) \sec ^{2} \beta_{1}\right. \\
& +\frac{1}{2} \sec \beta_{1}\left(\frac{a_{1}^{2}}{a^{2}}+\frac{c^{2}}{a^{2}} \sec ^{2} \beta_{1}\right)^{1 / 2}+\left(1-\frac{c}{a}\right) \operatorname{cosec}^{2} \alpha+\frac{1}{2} \operatorname{cosec} \alpha\left(\frac{a_{1}^{2}}{a^{2}}+\frac{c^{2}}{a^{2}} \operatorname{cosec}^{2} \alpha\right)^{1 / 2} \\
& \left.+\left(1-\frac{c}{2 a}\right) \sec ^{2} \beta\right\}+\frac{a_{2}}{a} \tan \beta\left\{1-\frac{c}{a}+\frac{1}{2}\left(\frac{h^{2}}{a^{2}}+\frac{c^{2}}{a^{2}}\right)^{1 / 2}\right\} \\
& +\left(\tan \beta+\frac{a_{2}}{a} \cot \alpha\right)\left\{1-\frac{c}{a}+\frac{1}{2}\left(\frac{h^{2}}{a^{2}}+\frac{c^{2}}{a^{2}}\right)^{1 / 2}\right\} .
\end{aligned}
$$


The symbols $c, a_{1}, \beta, \beta_{1}, \gamma$ have the meanings designated in Fig. 4, while $a_{2}, \alpha, \alpha_{1}, \delta$ are obtained from the relations $a=a_{1}+a_{2}, \cot \alpha+\tan \beta=a_{1} / h, \cot \alpha_{1}+\tan \beta_{1}=$ $a_{2} / h$, cot $\delta+\tan \gamma=c / h$. By Theorem 2, the function $g$ is an upper bound for the collapse value of the average indentation pressure, and, for a particular value of $a / h$, the best upper bound is obtained by minimising the function $g$ with respect to the variables $c, a_{1}, \beta, \beta_{1}, \gamma$. The minimum values of the function $g$ were obtained approximately by a process of trial and error for certain values of $a / h$. The values of the upper bound $p_{u}$ obtained and the values of the variables used in each case are shown in the following table. It is thought that the exact minimum values of $g / k$ do not differ from the values of $p_{u} / k$ shown by more than one unit in the last decimal place.

$\begin{array}{rccccc}a / h: & 3.5 & 4 & 5 & 5.5 & 6 \\ p_{u} / k: & 6.19 & 6.34 & 6.72 & 6.94 & 7.17 \\ c / a: & 0.62 & 0.58 & 0.54 & 0.52 & 0.50 \\ a_{1} / a: & 0.46 & 0.46 & 0.48 & 0.48 & 0.50 \\ \beta: & 21^{\circ} & 24^{\circ} & 31^{\circ} & 34^{\circ} & 36^{\circ} \\ \beta_{1}: & 25^{\circ} & 28^{\circ} & 33^{\circ} & 36^{\circ} & 36^{\circ} \\ \gamma: & 28^{\circ} & 30^{\circ} & 34^{\circ} & 34^{\circ} & 36^{\circ}\end{array}$

The curve marked III in Fig. 2 joins the values of $p_{u}$ plotted against $a / h$.

The velocity field of Fig. 4 was further modified by increasing the number of blocks of material under the area $O A B D$ from four to six and by increasing the number of blocks under the area $D B C$ from two to four. The resulting field provides an upper bound which is a function of nine variables. In order to simplify the work, the blocks under the areas $O A B D$ and $D B C$ were taken to have equal widths in the $O A$ and $D C$ directions respectively, and corresponding angles were taken to be equal. The upper bounds $7.18 k$ and $7.57 k$ were obtained for the cases $a / h=6$ and $a / h=7$ respectively. The bounds are not the best that can be obtained from this type of velocity field, but it was thought that any further lowering of the bounds would not justify the numerical work involved. The two bounds are plotted in Fig. 2 and joined by a straight line (marked IV in the figure).

Finally, we describe a velocity field which can be used to give reasonable upper bounds for the indentation pressure for large values of $a / h$. In Fig. 5, LMNO is the square area of indentation on the surface of the layer and as before the initial downward velocity of this area is taken to be $v$. The field has four vertical planes of symmetry, two passing through the mid-points of opposite sides of the square and two passing through opposite corners of the square. Because of this symmetry, it is only necessary to consider that part of the velocity field which accommodates the downward motion of the triangle $C B N$. The triangle $D E H$ is the vertical projection of the triangle $C B N$ on the lower surface of the layer. $B E F G H N$ is part of a right circular cylinder with axis $B N, B A F G P N$ is a right triangular prism, and angle $F A B$ is denoted by $\alpha$. In region $C B N H E D$ (and in fact in the whole of the material lying vertically below the square $L M N O$ ), the velocity components referred to the axes shown in the figure are taken to be

$$
V_{x}=\frac{x v}{2 h}, \quad V_{y}=\frac{y v}{2 h}, \quad V_{z}=-\frac{z v}{h} .
$$

These components satisfy the incompressibility condition (4), and satisfy the condition that the downward velocity of $C B N$ be $v . D E H$ is a surface of discontinuity between 
the material flowing in $C B N H E D$ and the material at rest immediately adjacent to the base. The discontinuity in velocity across this surface is, from (10), given by

$$
\Delta v=\frac{v}{2 h}\left(x^{2}+y^{2}\right)^{1 / 2} .
$$

The rectangle $B E H N$ is also a surface of velocity discontinuity. The flow in the regiom $B A F E H G P N$ is a plane strain motion with streamlines parallel to the line $E F A$. The velocity has the constant value $a v / 2 h$ along the streamlines in order that the normal velocity be continuous across $B E H N$. The jump in tangential velocity across this surface is of amount

$$
\Delta v=\frac{v}{2 h}\left(y^{2}+4 z^{2}\right)^{1 / 2} .
$$
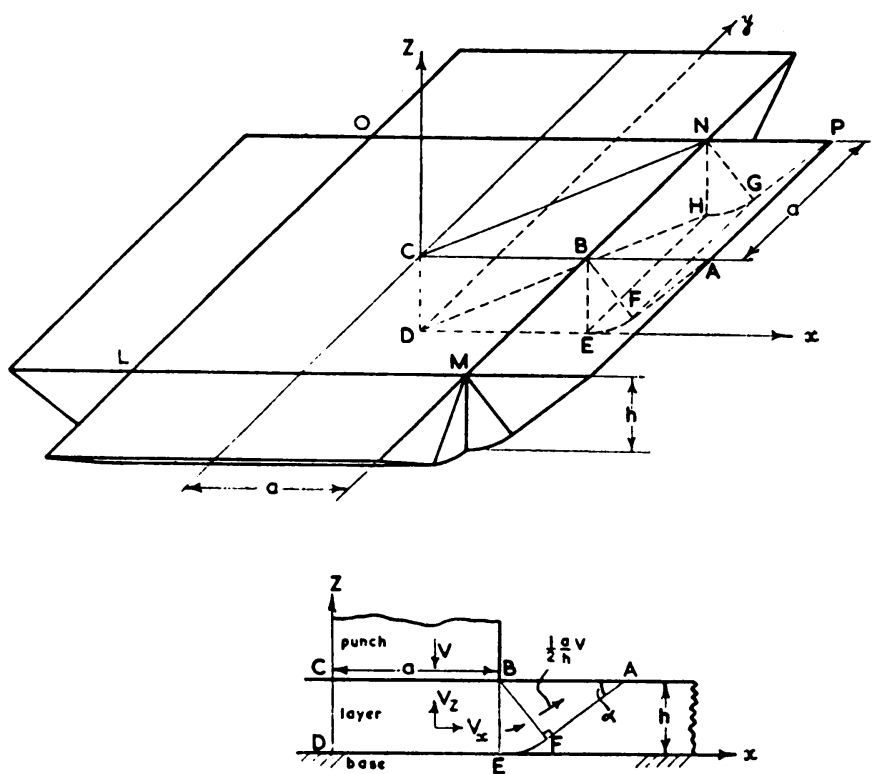

VERTICAL SECTION THROUGH CB.

FIG. 5. Velocity field for wide square punch.

Energy is dissipated in the discontinuity surfaces $D E H, B E H N, H P N$, and $E F A P G H$. Energy is also dissipated in the material vertically below $C D N$ and in the cylindrical region $B F E H G N$ due to non-zero plastic strain rates. After a little calculation, it is found that the velocity field will be kinematically admissible if the average pressure on the punch is not less than $k$ times the expression

$$
\begin{aligned}
j(\alpha)=2+ & 2 \alpha+\cot \alpha+\frac{h}{2 a}(\alpha+\cot \alpha)+0.383 \frac{a}{h}+\frac{1}{3}\left(1+4 \frac{h^{2}}{a^{2}}\right)^{1 / 2} \\
& +\frac{a}{12 h} \log _{\odot}\left\{2 \frac{h}{a}+\left(1+4 \frac{h^{2}}{a^{2}}\right)^{1 / 2}\right\}+\frac{2}{3} \frac{h^{2}}{a^{2}} \log _{\odot}\left\{\frac{a}{2 h}+\left(1+\frac{a^{2}}{4 h^{2}}\right)^{1 / 2}\right\} .
\end{aligned}
$$


By Theorem $2, k j(\alpha)$ is an upper bound for the collapse value of the average pressure over the punch. The function $j(\alpha)$ has a minimum value with respect to $\alpha$ when

$$
\sin \alpha=\frac{(2 a+h)^{1 / 2}}{(4 a+h)^{1 / 2}} \text {. }
$$

The upper bounds $p_{u}$, given by the minimum values of $k j(\alpha)$, are plotted in Fig. 2 for values of $a / h$ between 5 and 7 (curve marked $V$ in the figure), in order to compare the bounds with those obtained above.

For very large values of $a / h$, the value of $\alpha$ given by (12) tends to 45 degrees. Neglecting the first and higher powers of $h / a$ and taking $\alpha=\pi / 4$ in (11), we find that for large values of $a / h$, the upper bound is given by

$$
p_{u}=k\left(5.07+0.383 \frac{a}{h}\right) .
$$

Upper bounds for the average collapse pressure under a rectangular punch could be obtained by modifying the velocity fields of this section.

5. Square punch. Lower bounds for the indentation pressure. In this section lower bounds are obtained for the value of the average indentation pressure over the square area of contact. The statically admissible stress fields employed are extensions into three dimensions of two-dimensional stress fields which can be used to give lower bounds for the collapse pressure in the corresponding plane strain problem.

In Fig. 6(a), $O H$ is the centre-line of the punch $O A$ indenting the upper surface of

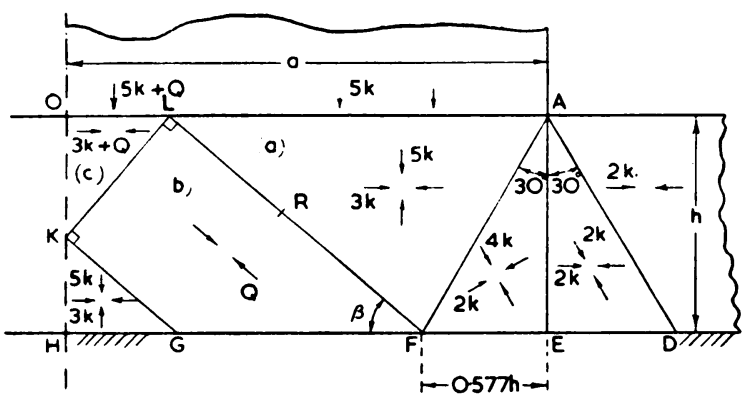

Fig. 6(a). Stress field for the plane strain problem.

the layer of thickness $h$ under conditions of plane strain. A pressure $5 k$ is produced on the surface of the punch by the stress system to the right of the line $A F$. This stress field nowhere violates Tresca's yield condition and was used previously in [2] to obtain the lower bound $5 k$ for the average indentation pressure in the indentation of an infinitely thick layer. The value $5 k$.is also a lower bound for a layer of finite thickness. The lines $A D, A E$, and $A F$ in Fig. 6(a) are lines of stress discontinuity and $A D, A F$ are each inclined to $A E$ at an angle of 30 degrees. The stresses in the different regions are shown in the figure.

In order to increase the lower bound, the pressure near the centre of the punch is increased. This is effected by superimposing a pressure $Q$ in the direction $F L$ on the stresses in the region $F G K L$. $F L$ is then a line of stress discontinuity, and the line $K L$ of stress discontinuity separates the stress system in $F G K L$ from the stress system in $O K L$ whose principal axes of stress are in the vertical and horizontal directions. We 
suppose that the regions $F G K L$ and $O K L$ are on the point of yielding in order to increase the pressure on $O L$ by as much as possible. Fig. 6(b) shows the Mohr's circles for regions $A F L, F G K L$, and $O K L$, which are denoted by a, b, c respectively. The circles a, b for regions a, $\mathrm{b}$ intersect at the stress point $R$ which gives the stress acting on a line element parallel to $F L$ in regions $\mathrm{a}$ and $\mathrm{b}$. The pole $P_{\mathrm{b}}$ of circle $\mathrm{b}$ is the intersection of the line

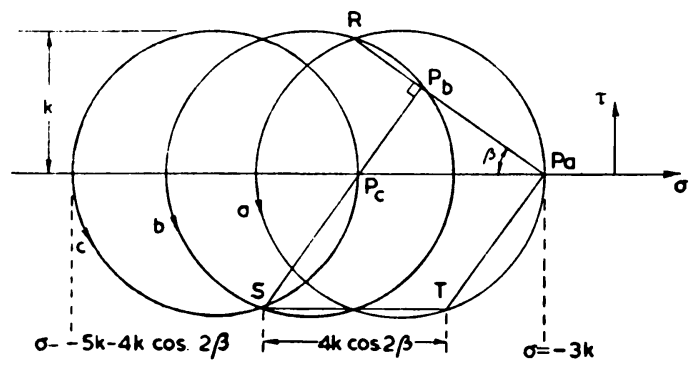

Fig. 6(b). Mohr's circles for regions (a), (b), (c) in Fig. 6(a).

joining $R$ to $P_{a}$, the pole of circle a, with circle $b$. The stresses acting on line elements perpendicular to $F L$ in regions a and $\mathrm{b}$ are given by the stress points $T$ and $S$ respectively, and the geometry of the figure shows that the value of $Q$ is $4 k \cos 2 \beta$, where $\beta$ is the angle $G F L$ in Fig. 6(a). ( $\beta$ must be less than 45 degrees in order that $Q$ be positive). Further, the circles b, c intersect at the stress point $S$ since the pole $P_{c}$ of circle c must lie on the $\sigma$-axis. The line of stress discontinuity $K L$ in Fig. 6(a) is therefore perpendicular to the line $F L$.

The pressure on $O L$ has the value $5 k+4 k \cos 2 \beta$ and the average pressure $p$ over $O A$ is found to be given by

$$
\frac{p}{k}=\tilde{j}+4 \cos 2 \beta\left\{1-\frac{h}{a}(0.577+\cot \beta)\right\},
$$

provided that $a_{\prime}^{\prime} h>1.577$, since $\beta<\pi / 4$. The stress field is statically admissible and, by Theorem 1, this is a lower bound for the collapse value of the average indentation pressure. For a given value of $a / h$ greater than 1.577, the lower bound is found by maximising (14) with respect to $\beta$. For values of $a / h$ less than 1.577 the lower bound is $5 k$. For values of $a / h$ greater than 4 approximately, it is better to repeat this process a certain number of times, as shown in Fig. 7, so that the pressure distribution on the

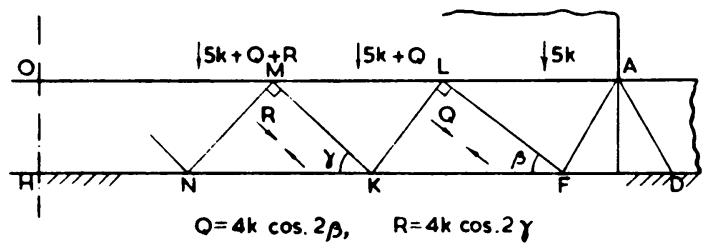

FIG. 7. Stress field for wide punch (plane strain).

punch has more than one jump in value. By taking the lines of discontinuity $L F, M K, \cdots$ in Fig. 7 to be inclined at an angle of $22 \frac{1}{2}$ degrees to the horizontal, the lower bound is found to he given by

$$
\frac{p}{k}=3.42+.24 \frac{h}{a}+\frac{1}{2} \frac{a}{h}
$$


provided that

$$
\frac{a}{h}=2.991+2.828 n,
$$

where $n$ is the number of lines of discontinuity $L F, M K, \cdots$. This is a good lower bound for large values of $a / h$.

Considering now the three-dimensional problem, a statically admissible stress field has been given previously [2] for the indentation of an infinitely thick layer by a square punch. This stress field shows that $5 k$ is a lower bound for the average collapse pressure and the bound also applies to the layer problem considered here. In the material just below the area of contact, the stress field involves a vertical compression of amount $5 k$ and an all-round horizontal compression of amount $3 k$. This state of stress extends throughout the material in the right pyramid formed by the square area of indentation and four triangular faces inclined at 30 degrees to the vertical, the vertex of the pyramid being vertically below the centre of the square. In obtaining lower bounds for the layer problem we shall assume that the material of the layer which lies in the pyramid is stressed in this way. The pressure near the center of the punch will be increased by a method similar to that used above in the plane strain problem.

Since the stress field is symmetrical about the two vertical planes passing through the mid-points of opposite sides of the square, we need only consider that part of the stress field which supports the pressure on one-quarter of the punch. The stress field is also symmetrical about the vertical planes through the diagonals of the square. In Fig. 8, $O A B C$ is one-quarter of the area of indentation in the upper surface of the layer, $O$ being the centre of the square area. For clarity, the points $A, B, C$, which are shown in the plan, are omitted from the perspective in Fig. 8. The triangular faces of the pyramid mentioned in the previous paragraph meet the lower surface of the layer in the lines $F X, R X$. The planes $L F W T, N R V T$ are each inclined at an angle $\beta$ to the horizontal, and the planes $L K P T, N M P T$ are perpendicular to the planes $L F W T, N R V T$ respectively.

As mentioned above, the stress field of [2] involves a vertical compression $5 k$ and an all-around horizontal compression $3 k$ in the material in the volume $O A B C R H F X$. For the moment we assume that the material vertically above the area $H F W V R$ is stress free. An all-around horizontal compression $3 k$ will be added later in this region in order to restore equilibrium. In the final stress field the stresses in the vertical plane through the line $O A$ will be as shown in Fig. 6(a). [In Fig. 6(a), the line $O H$ intersects the line $L K$, which is not the case in Fig. 8. However, if the width of the punch is such that $O H$ intersects $L K$ in Fig. 8, a simple adjustment can be made to the stress field in order that the symmetry conditions be satisfied, as they are in Fig. 6(a)]. The minimum principal stress in region b of Fig. 6 (a) is $3 k+2 k \cos 2 \beta$ so that, to satisfy the yield condition, the pressure parallel to $L T$ in region $L F W T P K$ of Fig. 8 must have this value at least.

We assume, therefore, a compression $2 k \cos 2 \beta$ parallel to $L T$ in region $L F W T P K$ and similarly a compression $2 k \cos 2 \beta$ parallel to $N T$ in region $N R V T P M$. The planes $T W P, T V P$ are surfaces of stress discontinuity and we assume that the stress system in region $T W V P$ is composed of two equal compressions of amount $S$ in the directions $T W$ and $T V$, so that the plane $T V W$ is stress free. From the equilibrium conditions across $T W P$ (and $T V P$ ), it is found that $S$ must have the value

$$
S=2 k \cos 2 \beta /\left(1+\sin ^{2} \beta\right) \cos ^{2} \beta,
$$


and that a vertical compression of amount

$$
T=2 k \cos 2 \beta \sec ^{2} \beta
$$

must be added in the material vertically above the planes $T W P, T V P$.

Compressions of amount $4 k \cos 2 \beta$ are now added in regions $L F W T P K$ and NRVTPM parallel to $F L$ and $R N$ respectively. Equilibrium is satisfied across the planes $L K P T$, $N M P T$ by adding a hydrostatic pressure of amount $4 k \cos 2 \beta$ in region OLTNMHKP. An all-around horizontal compression $3 k$ is now added throughout the material vertically
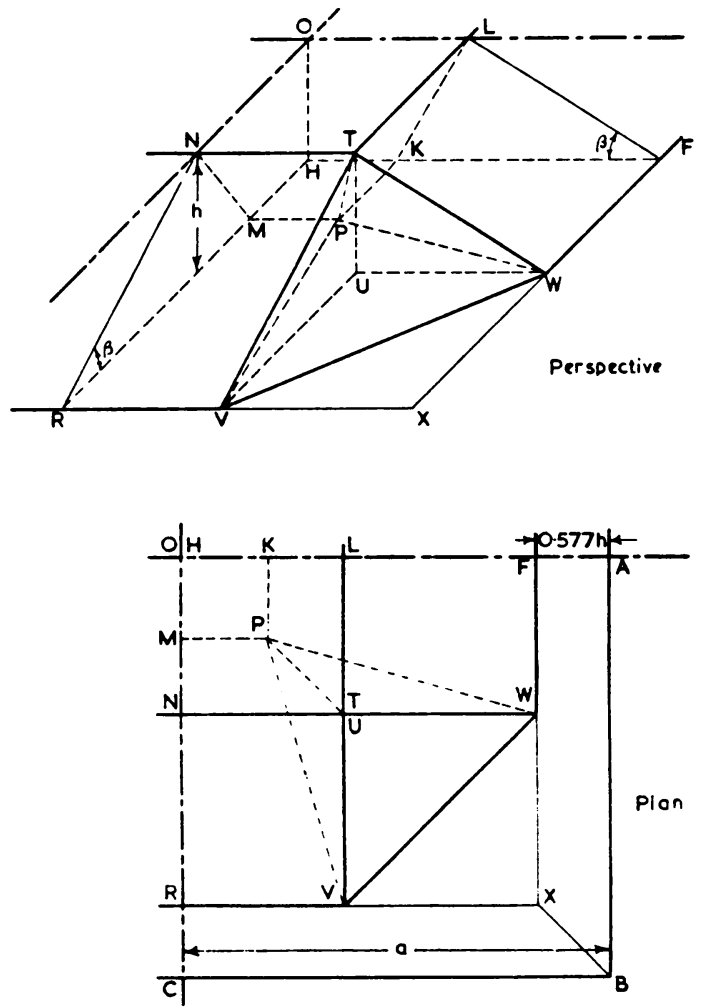

FIG. 8. Stress regions in statically admissible stress field for square punch.

above the area $H F W V R$. Vertical compressions of amount $5 k$ in the region vertically above the area $H F W P V R H$ and amount $5 k-T$ in the region vertically above the area $P W U V$ are added, and it can be verified that the yield condition is not violated in these regions. Above the area $U V W$ it is found that the maximum vertical compression which can be added without violating yield is given by $u(\beta)$, where $u(\beta)$ is the lesser of $5 k$ and $k$ times the expression

$$
3+2\left\{1-\frac{2 \cos ^{2} 2 \beta \sin ^{2} 2 \beta}{\left(1+\sin ^{2} \beta\right)^{2} \cos ^{4} \beta}\right\}^{1 / 2}+\frac{2 \cos 2 \beta\left(\cos 2 \beta-\sin ^{2} \beta\right)}{\left(1+\sin ^{2} \beta\right) \cos ^{2} \beta} .
$$

When $\beta$ is less than 25 degrees, $u(\beta)$ has the value $5 k$.

The total force on the area $O A B C$ of the punch due to this stress system is the resultant 
of a pressure $5 k$ over the area $T L A B C N$ less the triangular area vertically above $U V W$, a pressure $5 k+4 \cos 2 \beta$ over the square area $O L T N$, and a pressure $u(\beta)$ over the triangular area vertically above $U V W$. The average pressure $p$ on the punch is found, for $a / h>1.577$, to be given by

$$
\frac{p}{k}=5+4 \cos 2 \beta\left\{1-\frac{h}{a}(0.577+\cot \beta)\right\}^{2}-\frac{1}{2} \frac{h^{2}}{a^{2}} \cot ^{2} \beta\{5-u(\beta) / k\} .
$$

Since the stress field is statically admissible, this value of $p$ is a lower bound for the average indentation pressure, by Theorem 1 . For values of $a / h$ less than 2 , maximising (15) with respect to $\beta$ does not give a lower bound greater than $5 k$. Lower bounds $p_{L}$ for the average collapse pressure for the cases $a / h=2.5,3,3.5,4,4.5,5$ were obtained by maximising (15) with respect to $\beta$. The bounds are plotted against $a / h$ in Fig. 2 and joined to the line $p_{L}=5 k(a / h<2)$ by a smooth curve, which is marked (i) in the figure. For values of $a / h$ greater than 4.5 approximately, it is better to increase the pressure near the centre of the punch by repeating this process a suitable number of times, as in the plane strain case. Lower bounds for the cases $a / h=5,6,7$ were calculated by repeating the process once and maximising with respect to the angles $\beta ; \gamma$ of Fig. 7 . The results are given in Fig. 2, where they are joined by the curve marked (ii).

When the process of increasing the pressure towards the centre is carried out $n$ times, where the $n$ angles $\beta, \gamma, \cdots$ are taken to be $22 \frac{1}{2}$ degrees, the lower bound is found to be

$$
p_{L}=k\left(3.42+\frac{1}{3} \frac{a}{h}+1.82 \frac{h}{a}-0.26 \frac{h^{2}}{a^{2}}\right)
$$

for $a / h=2.991+2.828 n$. For very large values of $a / h$, the difference between the upper bound (13) and the lower bound (16) is 15 per cent of the lower bound, and the true value of the average pressure is given by $0.358 \mathrm{ka} / \mathrm{h}$ to within $\pm 7 \%$.

We now describe a stress field which provides better lower bounds than those obtained above for values of $a / h$ less than 4 approximately. The improvement in the lower bounds is less than $2.84 \%$ and is therefore not of great interest insofar as numerical results are concerned. However, it is thought that the result that, in the indentation of an infinitely thick layer, the average indentation pressure over a square punch is not less than the average indentation pressure over a long rectangular punch (plane strain), is of sufficient interest to justify its inclusion in this paper. The stress field used is an extension into three dimensions of a stress field previously used [6] to show the validity of the Prandtl stress solution [7] to the plane strain indentation of a layer of infinite thickness. This stress field is shown in Fig. 9, in which $O D$ is the centre-line of the punch $O A$ indenting the material below the line $O F$. The region $O F E D$ is composed of the Prandtl stress field of two regions of constant state, $A F E$ and $A D O$, and a zone of radial shear $A E D$. The Prandtl field is continued to the line of stress discontinuity $D S H$. The stress field below this line is assumed to satisfy the following conditions. (i) $\sigma_{x}$ and $\sigma_{z}$ are functions of $z$ and $x$ only respectively and $\tau_{x}$ is zero, so that the equilibrium equations (1) and the symmetry conditions across the centre-line $D R$ are satisfied. (ii) Immediately below the line of discontinuity, the material is in a plastic state of stress. These conditions and the equilibrium conditions across the line of discontinuity are sufficient to determine the stresses in the region DSHRD and to determine the line DSH. It is found that

$$
\left.\begin{array}{l}
-\sigma_{x}(\alpha) / k=2+\pi / 2+2 \alpha-2 \cos (\pi / 4-\alpha), \\
-\sigma_{z}(\alpha) / k=\pi / 2+2 \alpha-2 \cos (\pi / 4-\alpha),
\end{array}\right\}
$$


where $\alpha$ is the angle $G A S$, measured clockwise. The angle of inclination $\psi$ of the line of discontinuity to the negative $x$-axis at the point $S$ is $3 \pi / 8-\alpha / 2$. Denoting the lengths of the lines $A D$ and $A S$ by $r_{0}$ and $r$, the line of discontinuity is found to be given by

$$
r / r_{0}=\frac{1}{2} \operatorname{cosec}^{2}(\pi / 8+\alpha / 2) \text {. }
$$

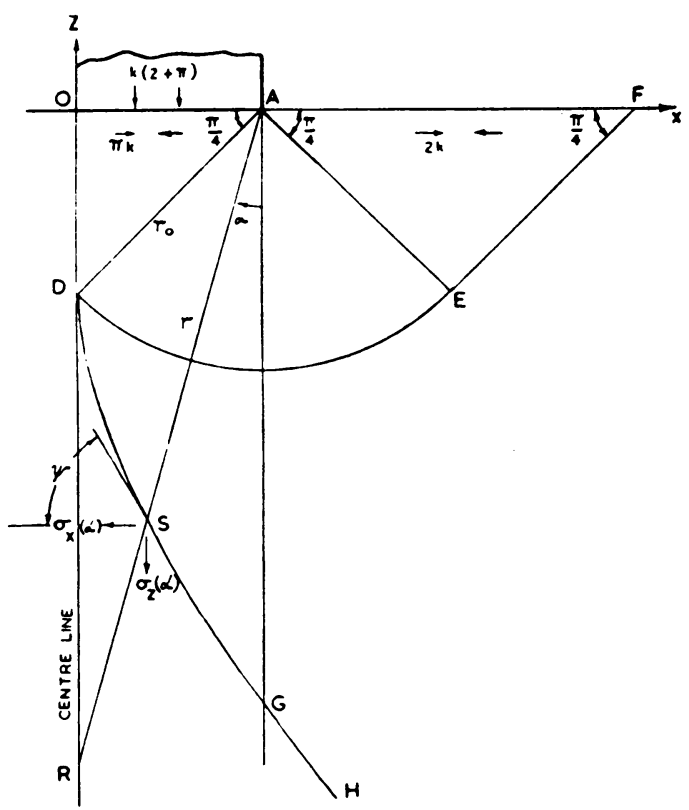

FIG. 9. Extension of Prandtl stress field for two-dimensional punch.

As $\alpha$ tends to the value $-\pi / 4$, the line tends asymptotically to a straight line inclined at an angle $\pi / 4$ to the negative $x$-axis. The field is statically admissible and provides the lower bound $(2+\pi) k$ for the average collapse pressure.

Referring now to Fig. 10(a), $O$ is the centre of the square area of indentation on the plane surface of the material, and $A, C$ are the mid-points of the two sides of the square which meet at the corner $B$. The stress field is symmetrical about vertical planes through $O A$ and $O C$ and through $O B$. The pressure on the area of indentation is $(2+\pi) k$ and there is an all-around compression of amount $\pi k$ in region $O A B D$. The section of Fig. 10 (a) by the vertical plane through $O A$ is given by Fig. 9, and the stresses in this plane are the stresses of the field of Fig. 9. This stress system is reproduced, apart from the linear scale, in the vertical plane through a line such as $L M$, parallel to $O A$. This plane is shown in Fig. 10(b). Sections of Fig. 10(a) by planes parallel to the vertical plane through $O C$ are similar because of the symmetry about the vertical plane through $O B$. The surface generated by straight lines from the point $B$ to points on the curve $D S H$ is a conical surface of stress discontinuity, as is the surface [not shown in Fig. 10(a)] generated by straight lines from $B$ to the curve $D K$. The region vertically below the triangle $A B D$ is subjected to stresses $\sigma_{\nu}$ in the direction $O C$. These stresses, which are not dependent on the coordinate $y$, originate from the discontinuity surface through the curve $D K$. Thus points in the plane $A B R$, which is inclined at an angle $\alpha$ to the vertical, are acted upon by the stress $\sigma_{\nu}(\beta)$ given by

$$
-\sigma_{y}(\beta) / k=2+\pi / 2+2 \beta-2 \cos (\pi / 4-\beta),
$$




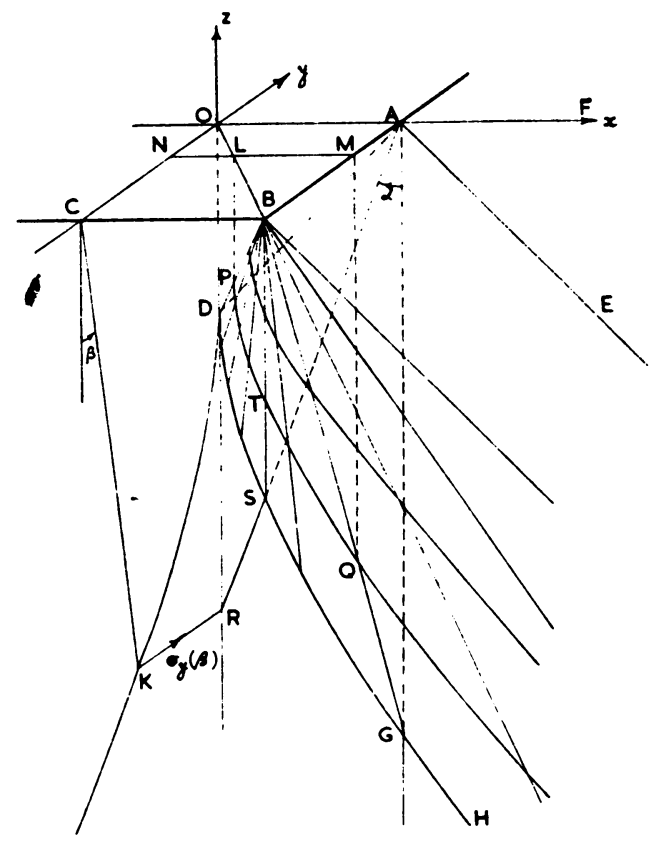

FIG. 10(a). Stress field for square punch on infinitely thick layer.

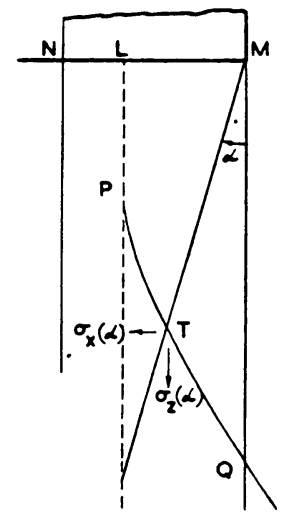

Fig. 10(b). Vertical section through $L M$ in Fig. 10 (a).

where $\beta$ is the inclination of $C K$ to the vertical and is given in terms of $\alpha$ by the relation

$$
\cot \alpha=\cos \beta /(2)^{1 / 2} \sin ^{2}(\pi / 8+\beta / 2),
$$

$K R$ being parallel to $C O$. This follows because $A R$ is the orthogonal projection of the generator $B K$ (not shown in the figure) on to the plane $O A G$, which is the plane $y=0$. As $\alpha$ decreases from $\pi / 4$ to zero, the value of $\beta$ given by (19) decreases from $\pi / 4$ to $-\pi / 4$. It can be verified that the stress system in the region vertically below the triangle $A B D$ does not violate the yield condition. In the same way the region vertically below the triangle $C B D$ is in a state of stress which does not violate the yield condition. In order to complete the stress field, a compressive stress of amount $2 k$ in the direction $A B$ is added to the region $x>a$, where $2 a$ is the width of the square, and a compression $2 k$ in the direction $B C$ is added to the region $y<-a$. The addition of these two com- 
pressions ensures that the yield condition is satisfied in the regions $x>a, y<-a$.

The field described is statically admissible and it follows from Theorem 1 that $(2+\pi) k$, or $5.14 k$, is a lower bound for the average collapse pressure. For a layer of finite thickness, the pressure near the centre of the punch can be increased by the same method used in the stress field associated with Fig. 8. The formula corresponding to (15) is found to be, for $a / h>2$,

$$
\frac{p}{k}=5.14+4 \cos 2 \gamma\left\{1-\frac{h}{a}(1+\cot \gamma)\right\}^{2}-\frac{1}{2} \frac{h^{2}}{a^{2}} \cot ^{2} \gamma\{5-u(\gamma) / k\},
$$

where $\gamma$ is the angle corresponding to angle $\beta$ in Fig. 8. Maximising (20) with respect to $\gamma$ does not give a lower bound greater than $5.14 k$ for $a / h$ less than 2.5 . The bounds $p_{L}$ obtained for the cases $a / h=3,3.5,4$ are plotted in Fig. 2 and joined to the line $p_{L}=5.14 k(a / h<2.5)$ by a smooth curve marked (iii) in the figure.

The broken line in Fig. 2 is obtained by averaging the least upper bound and the greatest lower bound for the collapse pressure for values of $a / h$. The upper and lower bounds show that this mean value curve determines the collapse pressure to within $\pm 5 \%$ for $a / h$ less than 2 , the possible error increasing to $\pm 10 \%$ for $a / h=7$.

The stress fields used in this section for the square punch can be adjusted in a simple manner in order to give lower bounds for the indentation pressure under a rectangular punch.

6. Circular punch. In this section upper and lower bounds are found for the average collapse pressure in the indentation of the layer by a flat circular punch. The bounds are close for layers which are not too thick in comparison with the width of the punch.

Fig. 11 shows a simple velocity field which satisfies the boundary conditions on

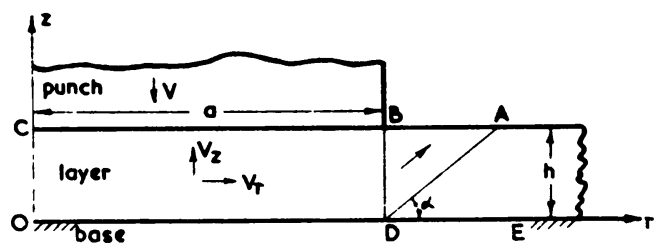

FIG. 11. Velocity field for circular indenter.

kinematically admissible fields. The field is symmetrical about the $z$-axis which passes through the centre of the punch. In the material vertically below the area of indentation, region $B C O D$ in Fig. 11, the radial and vertical components $V_{r}, V_{z}$ of velocity are taken to be $V_{r}=V r / 2 h, V_{s}=-V z / h$, where $V$ is the downward velocity of the punch. These components represent an incompressible flow. In the remainder of the velocity field, region $A B D$, the streamlines are parallel to the conical surface $A D$, which is a surface of velocity discontinuity. The velocity conditions across the discontinuity surface $B D$ together with the incompressibility condition determine the velocity components in region $A B D$ to be given by $V_{r}=V a^{2} / 2 r h, V_{s}=V a^{2} \tan \alpha / 2 r h$, where $\alpha$ is the angle $A D E$. A straightforward calculation shows that the velocity field will be kinematically admissible if the average pressure on the punch is greater that $k q(\alpha)$, where

$q(\alpha)=2+\frac{h}{a}+\frac{1}{3} \frac{a}{h}+(1+\sec \alpha)\left\{\left(1+\frac{a}{h} \tan \alpha\right) \log _{e}\left(1+\frac{h}{a} \cot \alpha\right)-1\right\}$

$$
+\operatorname{cosec} \alpha \sec \alpha+\tan \alpha \text {. }
$$


Upper bounds $p_{u}$ are obtained by minimising (21) with respect to $\alpha$ for particular values of $a / h$. For $a / h=7$, for example, the minimum value of $q(\alpha)$ is near $\alpha=36^{\circ}$ where it has the value 7.51. If the first and higher powers of $h / a$ are neglected in expression (21) for $q(\alpha)$ we obtain

$$
q(\alpha)=2+\frac{1}{3} \frac{a}{h}+\operatorname{cosec} \alpha \sec \alpha+\tan \alpha .
$$

This expression has a minimum value for $\alpha=35^{\circ} 16^{\prime}$ so that, for large values of $a / h$,

$$
p_{u}=k\left(4.83+\frac{1}{3} \frac{a}{h}\right)
$$

is an upper bound for the collapse value of the average identation pressure.

Attempts were made to extend the stress field of Fig. 6(a) to a circular area of indentation. It proved to be a matter of some difficulty to provide the appropriate stresses normal to the plane of Fig. 6(a) (i.e. the circumferential or hoop stresses) which are required by the yield criterion. An alternative two-dimensional stress field, which can be extended to the circular punch problem in a simple manner, is shown in Fig. 12(a).

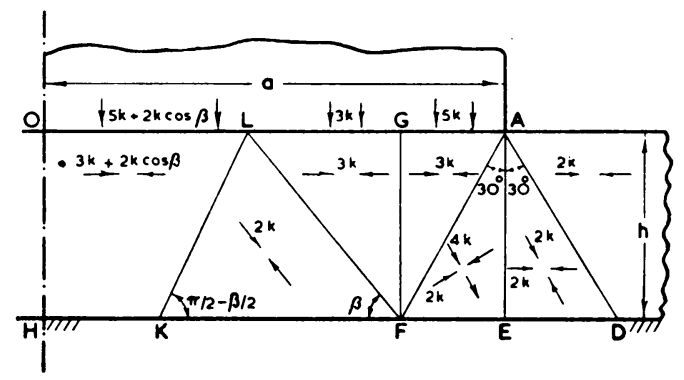

FIG. 12(a). Alternative stress field for the plane strain problem.

The field to the right of the line $A F$ produces a pressure $5 k$ on the portion $A G$ of the punch, as in Fig. 6(a). In region $F G L$ the state of stress is a hydrostatic pressure of amount $3 k$, and in region $F K L$ a compressive stress $2 k$ in the direction of $F L$ is added to the hydrostatic pressure $3 k$. The discontinuity line $L K$ is inclined at an angle $\beta / 2$ to the vertical, where $\beta$ is the angle $K F L$, and a pressure $5 k+2 k \cos \beta$ is produced on $O L$. The field is statically admissible and provides lower bounds for the indentation pressure. These lower bounds are less than those obtained from the stress field of Fig. 6(a).

As mentioned in [2], the field to the right of the line $A F$ in Fig. 12(a) can be modified so that it applies to any convex area of indentation and to a circular area in particular. The modified field lies outside the conical surface through $A F$, shown in plan in Fig. 12 (b). Vertically above the conical surface through $A F$, the material is subjected to a vertical compression $5 k$ and radial and circumferential compressions $3 k$. The material lying between the cylindrical surface through $G F$ and the conical surface through $L K$ is stressed by a hydrostatic pressure $3 k$. In addition, "legs" of material in this region, originating from strip elements of area on the conical surface through $L K$ and inclined at an angle $\beta$ to the horizontal, carry compressions of amount $2 k$. Thus the trapezium $L K K^{\prime} L^{\prime}$ is a strip element of area on the conical surface through $L K$ subtending an angle $\delta \theta$ at the axis $O H$ and generating the "leg" $L K F F^{\prime} K^{\prime} L^{\prime}$ which carries a compression 
$2 k$ in the direction $F L$. The material inside the conical surface through $L K$ is stressed by a vertical compression $5 k+2 k \cos \beta$ and equal radial and circumferential compressions of amount $3 k+2 k \cos \beta$. Triangular elements of area, such as $L^{\prime} F^{\prime} M$ in the plan, on the area of indentation in the annulus bounded by the circles through $L$ and $G$ do not lie vertically above the "legs" of material. The pressure over these areas can be in-

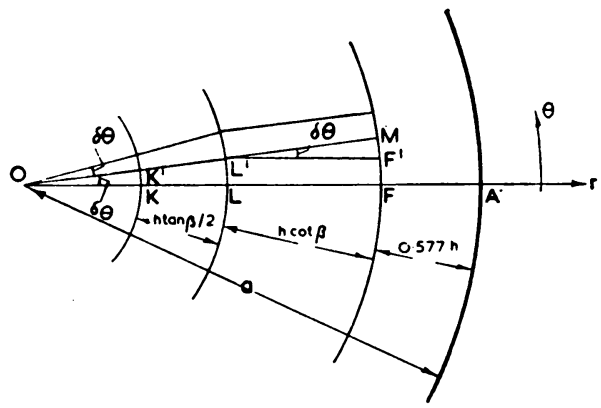

Fig. 12(b). Plan of stress regions in field for circular punch.

creased from $3 k$ to $5 k$ by adding a vertical compression $2 k$ in the triangular prisms of material below the areas. The field described is statically admissible since it does not violate Tresca's yield criterion and satisfies the equilibrium equations and the boundary conditions. It therefore provides a lower bound for the average collapse pressure. For layers whose thicknesses are not too large compared with the width of the punch, the process of increasing the pressure towards the centre of the punch can be repeated. Thus when $a / h=1.577+1.414 n$, where $n$ is an integer, the average pressure over the punch is found to be

$$
p_{L}=k\left(2.72+\frac{1}{3} \frac{a}{h}+3.05 \frac{h}{a}-0.44 \frac{h^{2}}{a^{2}}\right),
$$

where the $n$ angles such as angle $\beta$ in Fig. 12(a) are taken to be 45 degrees. The percentage difference between the lower bound (23) and the upper bound (22) tends to zero as $a / h$ tends to infinity.

The stress field used here for a circular punch can be modified so that it applies to any area of contact which is convex. It can therefore be used to provide lower bounds for any convex area of indentation.

Acknowledgements. This work was begun in the course of research sponsored by the Armstrong Cork Co., Lancaster, Pa., while the author was a Research Associate in Applied Mathematics at Brown University, Providence, R. I. The work was completed at the author's present address and the author is indebted to the Armstrong Cork Company and to the Chief Scientist, Ministry of Supply, for permission to publish this paper.

\section{BibLIOGRAPHY}

(1) A. Wang and E. H. Lee, Plastic flow in a thin sheet of perfectly plastic material laid on a rough rigid base and compressed by a smooth flat disc, Report AC-47 to Armstrong Cork Co., Brown University, 1953.

(2) R. T. Shield and D. C. Drucker, The application of limit analysis to punch-indentation problems, J. Appl. Mech. 20, 453-460 (1953). 
(3) H. J. Greenberg and W. Prager, Limit design of beams and frames, Proc. Am. Soc. Civil Engrs. 77, Separate No. 59 (1951); Trans. A.S.C.E. 117, 447-484 (1952).

(4) D. C. Drucker, H. J. Greenberg and W. Prager, The safety factor of an elastic-plastic body in plane strain, J. Appl. Mech., 73, 371-378 (1951).

(5) D. C. Drucker, W. Prager and H. J. Greenberg, Extended limit design theorems for continuous media, Q. Appl. Math. 9, 381-389 (1952).

(6) R. T. Shield, Plastic potential theory and Prandtl bearing capacity solution, J. Appl. Mech. 21, 193-194 (1954).

(7) L. Prandtl, Ueber die Haerte plastischer Koerper, Nachrichten Ges. Wiss. Goettingen, Math. Phys. K1. 1920, pp. 74-85 (1920). 